\title{
BMJ Open Efficacy of a topical herbal and mineral formulation (Dynamiclear) for the treatment of herpes simplex labialis in the community setting: study protocol for a randomised, double-blind placebo- controlled trial
}

\author{
Mike Armour (D) , ${ }^{1,2}$ Alex Semprini (D) , ${ }^{1,2}$ Carolyn Ee,${ }^{1}$ Lois MacCullagh, \\ Nick Shortt ${ }^{1,2}$
}

To cite: Armour M, Semprini A, Ee C, et al. Efficacy of a topical herbal and mineral formulation (Dynamiclear) for the treatment of herpes simplex labialis in the community setting: study protocol for a randomised, double-blind placebocontrolled trial. BMJ Open 2020;10:e031876. doi:10.1136/ bmjopen-2019-031876

- Prepublication history and additional material for this paper are available online. To view these files, please visit the journal online (http://dx.doi. org/10.1136/bmjopen-2019031876).

Received 13 June 2019 Revised 27 November 2019 Accepted 12 December 2019

Check for updates

(C) Author(s) (or their employer(s)) 2020. Re-use permitted under CC BY-NC. No commercial re-use. See rights and permissions. Published by BMJ.

${ }^{1}$ NICM Health Research Institute, Western Sydney University, Penrith, New South Wales,

Australia

${ }^{2}$ Medical Research Institute of New Zealand, Wellington, New Zealand

Correspondence to Dr Mike Armour;

M.Armour@westernsydney. edu.au

\section{ABSTRACT}

Introduction Herpes simplex labialis (HSL) is a common infection that can cause painful lesions on the oral mucosa, commonly referred to as cold sores. Current biomedical treatments include topical aciclovir, which reduces the episode duration by an average of 0.5 days. This study will examine the efficacy and tolerability of an over-the-counter topical treatment, Dynamiclear in reducing duration and severity of HSL episodes.

Methods and analysis This prospective, randomised, double-blind, placebo-controlled, multi-centre trial will recruit a minimum of 292 adult participants across Australia and New Zealand who present with a cold sore within 48 hours of onset. They will be randomly allocated in a 2:1 ratio to receive either topical Dynamiclear (active) or placebo. Dynamiclear's active ingredients are Hypericum perforatum, Calendula Officinalis and copper sulfate. A single topical treatment of active or placebo will be applied by a pharmacy-based investigator, and participants will be provided with a viral swab kit to confirm presence of herpes virus 1 or 2 from ulcerated lesions. Participants will receive reminders by email and/ or SMS to complete an online daily diary assessing their cold sore lesion using a visual guide, and recording other symptoms on numeric scales until healed. The primary outcome variable is median duration of HSL episode in days (participant evaluated) from presentation to return to normal skin. Secondary outcomes include severity of lesion pain, itching, burning and tingling during the symptomatic phase and proportion of lesions progressing to ulceration.

Ethics and dissemination Australian ethics approval from Western Sydney University Human Research Ethics Committee, ref: H12776. New Zealand Ethics approval from The Health and Disability Ethics Committees (HDEC) ref: $18 / C E N / 151$. Results will be published in a peerreviewed academic journal, presented at academic meetings and reported to participants

Trial registration numbers Australia and New Zealand Clinical Trials Registry (ACTRN12618000890235); Universal Trial Number (UTN) (U1111-1233-2426).
Strengths and limitations of this study

Novel pharmacy research network allows for effective recruitment of herpes simplex labialis sufferers that most often access treatment in the community.

- Single-dose intervention eliminates treatment compliance issues.

- Digital data collection minimises study burden for investigators and participants and ensures realtime, accurate data collection.

- Self-report measures for lesion progression verified by pharmacy visit 2 .

- Self-reported outcomes for days in-between pharmacy visits are subjective and may overestimate or underestimate lesion progression or symptom severity.

\section{INTRODUCTION}

Herpes simplex labialis

Herpes simplex labialis (HSL) is a common recurring infection of the labial or perioral skin caused by herpes simplex virus (HSV), also known as cold sores. The majority of cases are caused by HSV-1. Seroprevalence studies indicate that $60 \%-90 \%$ of adults are infected with HSV-1 and that HSV is the fastest growing infectious disease in the world with 500000 new cases reported each year. ${ }^{1}$ Approximately $20 \%-40 \%$ of the population experiences recurrent outbreaks of HSL. The frequency of outbreaks ranges from rare episodes every $5-10$ years, to monthly or more frequent outbreaks. ${ }^{2}$

Symptoms of active infection include prodromal numbness and tingling around the affected area, before progression to erythema, itching, burning, pain in the area and ulcerated lesions. Disease episodes are 
generally mild and self-limiting ${ }^{1}$; however, the symptoms are uncomfortable and often more importantly aesthetically unpleasant and noticeable to the general public.

Primary herpes simplex infection is most often asymptomatic, however can manifest significant morbidity including general anorexia and malaise, fever, local lymphadenopathy, gingivostomatitis and significant ulcerative lesions; these symptoms may persist for up to 3 weeks. ${ }^{3}$ Following the initial infection, the virus establishes a chronic, latent and lifelong infection in sensory nerve ganglia, predominantly the trigeminal ganglion. ${ }^{4}$ At a later date, the virus may be reactivated and travel back to the oral mucosa, perioral skin and/or labial surfaces, where it replicates, producing a clinical episode of recurrent herpes labialis. Viral reactivation usually occurs due to stress on the immune system. Triggers include exposure to ultraviolet (UV) light exposure, ${ }^{5}$ and viral infections such as colds and flu. ${ }^{6}$ Recurrent episodes are shorter than the initial episode, with the virus often cleared within 3 days or less, due to the previously acquired immune response. ${ }^{1}$ This immune response contributes to rapid control of recurrent HSL disease episodes but may also be responsible for clinical signs and symptoms such as pain, redness and swelling that can persist for up to a week or longer, even after the virus can no longer be isolated. $^{2}$

\section{Current standard treatments for HSL}

Oral and topical aciclovir preparations are used as the current standard of care for the treatment of HSV. Oral antiviral medications such as aciclovir, valaciclovir, famciclovir limit replication of the HSV by inhibiting viral DNA polymerase, and can shorten the time to healing from half a day to just over 2 days compared with placebo depending on the type of medication and dosage used. ${ }^{1}$ Adverse events are generally mild and similar to placebo. ${ }^{78}$

Topical treatment with aciclovir and other antivirals have also shown modest clinical benefit, reducing average healing time by around half a day compared with vehicle control. ${ }^{19}$ Topical aciclovir demands good compliance, requiring daily application but is generally well tolerated, with an adverse event rate similar to vehicle control. ${ }^{9}$

\section{Composition of Dynamiclear}

Dynamiclear (Sci-chem International) contains three active ingredients traditionally used to help treat inflamed or infected skin: Hypericum perforatum extract, Calendula officinalis extract and copper sulfate pentahydrate.

H. perforatum, or St. John's Wort, has demonstrated antiviral activities against enveloped viruses such as HSV and HIV. The active constituent, hypericin, reduces viral replication, through inhibition of new virion budding, prevention of viral uncoating and inhibition of protein kinase activity. ${ }^{10}$ Hypericin has also been found to inhibit protein kinase $\mathrm{C}$ gamma and epsilon, which are proteins associated with the development of neuropathic pain. ${ }^{10}$ For treatment of HSL skin lesions, $H$. perforatum is likely to be effective for topical application only as ingestion can cause photosensitivity, which may promote HSL reactivation. ${ }^{11}$

C. officinalis has been traditionally used for minor skin infections and inflammation. ${ }^{12}$ Recent studies have found calendula to be effective in reducing the time to healing in previously non-healing venous leg ulcers. ${ }^{13}$ This is likely to occur through the upregulation of genes controlling connective tissue growth factor and $\alpha$-smooth muscle actin ${ }^{14}$ and the proliferation and migration of fibroblasts. ${ }^{15}$ C. officinalis may therefore reduce the time to wound healing in HSL lesions that progress to an ulcerative phase.

Copper sulfate is a naturally occurring mineral which has demonstrable antiviral activity. Rather than suppressing viral replication, copper ions render the viral DNA nonviable for further replication. HSV has been shown to exhibit sensitivity to low concentrations of copper, and in vitro research has shown evidence for copper-mediated inactivation of HSV. ${ }^{16}$

A non-blinded, active comparator randomised controlled trial ${ }^{17}$ of a previous version of Dynamiclear (without C. officinalis) demonstrated lower odds of having pain, redness or progressing to the vesicle stage compared with topical $5 \%$ aciclovir in the treatment of orolabial and genital herpes simplex episodes. However, interpretation of these results is limited due to the inclusion of multiple subtypes of HSV, lack of blinding and use of non-standard outcome measures.

\section{Study aims}

Primary objective

To evaluate the efficacy of one topical dose of Dynamiclear in the treatment of HSL by a reduction in episode duration.

\section{Secondary objectives}

1. To assess the effect of Dynamiclear on the progression to ulcerative $\mathrm{v}$ non-ulcerative lesions.

2. To assess the effect of Dynamiclear on the duration of wound healing.

3. To assess the effect of Dynamiclear on pain, tenderness, tingling and itching during the disease course of HSL.

4. To assess patient satisfaction of the Dynamiclear product.

\section{Hypothesis}

A single topical application of Dynamiclear has greater efficacy than placebo on the severity and duration of HSL

\section{METHODS AND ANALYSIS \\ Study design}

A prospective, randomised controlled, double-blind, multi-site trial to evaluate the efficacy of a single dose of topically applied Dynamiclear versus placebo on the duration and severity of HSL. 


\section{Box 1 Inclusion and exclusion criteria}

\section{Inclusion criteria}

- Age 18-65 years.

- Onset of prodromal or clinical symptoms of HSL in the past 48 hours.

- Previous clinical history of HSL with at least three prior episodes of HSL.

- Primary lesion within $1 \mathrm{~cm}$ of the lip.

- Willing to provide informed consent and adhere to study protocol.

- Has internet (either by mobile or computer) to complete online forms.

\section{Exclusion criteria}

- History of immunodeficiency, immunosuppression or autoimmune disorder.

- Current infection not related to the study condition-for example, infections such as cold or influenza.

- Use of other antiviral, anti-inflammatory or steroid medications during or within 2 weeks prior to the treatment period.

- Use of other topical agents (including cosmetics, lip balms, sunscreens) or cosmetic procedures (such as chemical peels or microdermabrasion) on the prodromal or lesion area during the treatment period.

- Mechanical disruption (eg, scrubbing lancing or shaving) of the prodromal or lesion area during the treatment period.

- Pregnancy, lactation or planning to become pregnant in the following 14 days.

- Participation in another clinical trial within the previous 30 days.

HSL, herpes simplex labialis.

\section{Sample size calculation}

The sample size needed to demonstrate statistically and clinically significant efficacy was determined using the hypothesis that the mean episode duration in the treatment group will be 5.0 days, compared with 6.0 days in the placebo group. This is based on the review of previous studies of aciclovir versus placebo by Harmenberg $e t a l^{1}$ and a clinically significant difference of 1 day in episode duration. Given an alpha of 0.05 , power of $80 \%$ and an allocation ratio of 2:1 for treatment to placebo, 149 participants are required in the treatment group and 75 in the placebo group (224 in total). A recent study of kanuka honey versus aciclovir to treat cold sores, using the New Zealand Pharmacy based Research Network (PRN) for recruitment, reported an $11 \%$ dropout rate. However, because our study is placebo-controlled, we anticipate a higher dropout rate. Allowing for a 30\% dropout rate, 292 participants are required: 194 in the treatment group and 98 in the control group. This sample size will also power the study to detect a moderate effect size on the secondary outcome measures (Cohen's $\mathrm{d}=0.4$ ).

\section{Participants}

A total of 292 participants aged 18-65 presenting to participating pharmacies within 48 hours of onset of prodromal symptoms of HSL and who report at least three prior episodes of HSL will be recruited. Inclusion and exclusion criteria are presented in box 1 .
A PRN in both New Zealand and Sydney, Australia will be used to register sites for primary recruitment, screening and assessment.

Study centres: 10-15 pharmacies in Sydney, Australia, and 20-25 pharmacies in New Zealand.

\section{Patient and public involvement (PPI)}

There were no funds or time allocated for PPI so we were unable to involve patients in the study design. We will invite patients at the conclusion of the study to provide feedback on the burden of the intervention, and patients will be involved in developing the dissemination strategies for our findings.

\section{Recruitment and selection}

Four parallel recruitment pathways are planned in this study. First, social media will be used to advise participants of the trial, and refer them to a web page which outlines the inclusion and exclusion criteria and encourage them to present at participating pharmacies within 48 hours of the onset of a cold sore. Second, the New Zealand arm of the PRN has recently completed a different HSL study. ${ }^{18}$ The 920 participants who joined that study and consented to being contacted for further studies will be sent an email informing them of this study. Third, all participating PRN pharmacies will have promotional material in their pharmacies and individuals presenting to these pharmacies with a cold sore recurrence within the past 48 hours will be invited to enrol in the study. Fourth, TrialFacts, a paid recruitment service, will be used to advertise and screen for potential participants. Recruitment will begin in June 2019.

Regardless of recruitment pathway, on presentation at a participating pharmacy, potential participants will be screened and those eligible will be consented by pharmacists and pharmacy technicians. Recruitment staff will ensure that each participant is fully informed about the nature and objectives of the study and possible risks associated with participation.

\section{Randomisation}

After providing informed consent and satisfying the eligibility criteria, participants will be randomised in a 2:1 ratio to receive either active treatment with Dynamiclear $(n=194)$ or the placebo-controlled group $(n=98)$ using the Castor EDC platform. ${ }^{19}$ Randomisation will be performed using the Castor EDC platform by a researcher independent of the study team. To maintain pharmacist blinding when using a 2:1 ratio, boxes containing the intervention are prerandomised, and pharmacists are provided with a box code rather than a group allocation. A 2:1 ratio in favour of the active intervention was chosen to maximise recruitment rates as potential participants are actively seeking treatment when attending the pharmacy. Thus, a greater chance of being allocated active treatment is likely to increase recruitment and retention rates. Permuted block randomisation, with a block size of 6 or 9 will be 
Box 2 Composition of interventional product (Dynamiclear) and placebo

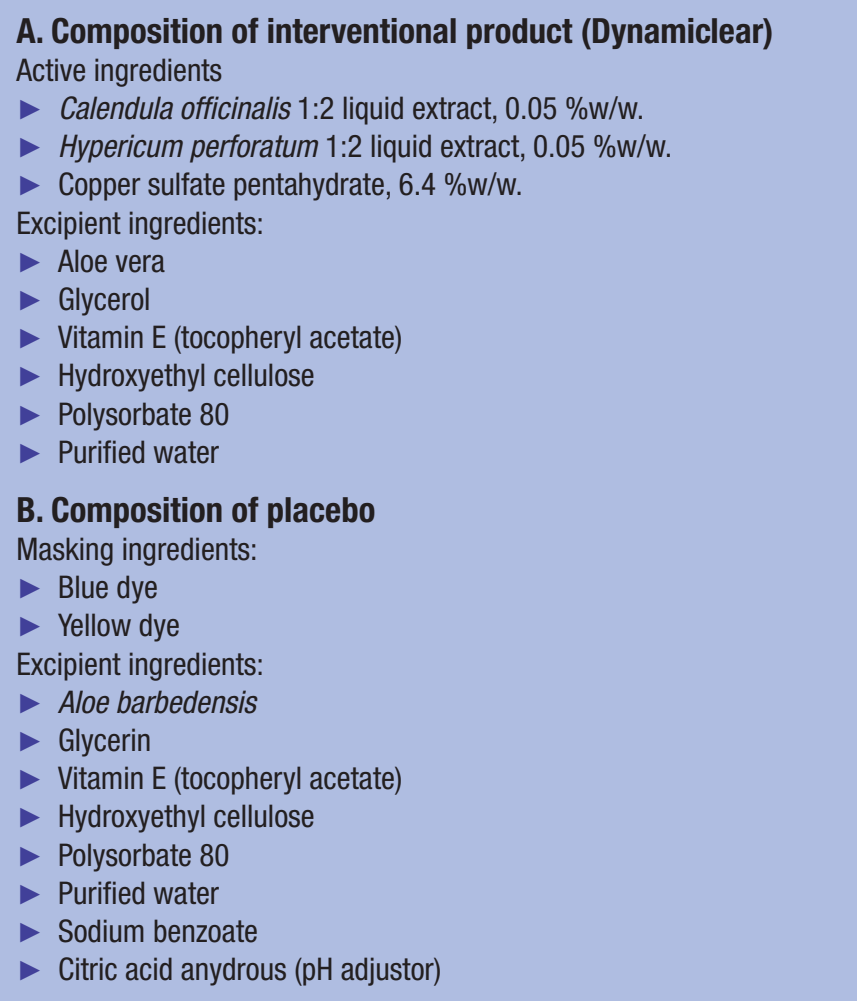

undertaken for each pharmacy to ensure that no patterns or imbalances emerge in group allocation between sites.

\section{Blinding}

All pharmacy staff, study team, participants and data analysts are blind to the group allocation. Unblinding will be permitted for either Dr Ee or Dr Semprini, or any other medical monitor, if serious adverse events are reported.

\section{Study interventions}

Interventional treatment is applied once only during pharmacy visit 1 . Each participant will be allocated one vial of Dynamiclear or a placebo that matches Dynamiclear in colour, viscosity, taste and smell. The active and placebo vials are presented in identical packaging. The intervention or placebo will be applied via a cotton-tipped applicator which delivers $0.7 \mathrm{~mL}$ to the affected area. This will be done either by the trained investigator or by the participant in view of the investigator, and the vial and packaging disposed of immediately. The moistened applicator will be applied for a minimum of $30 \mathrm{~s}$, directly on the HSL lesion. No investigational product or packaging will leave the pharmacy site for any reason. Box 2 outlines the composition of both the interventional product and placebo.

\section{Data capture and monitoring}

All data will be captured electronically via the secure electronic data capture platform CastorEDC. ${ }^{19}$ Each pharmacy will be provided with an iPad (Apple Inc) which will be used to collect all data during each face to face clinical visit. Signed consent forms will be digitally captured and uploaded directly onto the EDC platform.

Each participant will attend two clinical visits at PRN pharmacies. Visit 1 will be within 48 hours of symptom onset and will include initial clinical assessment and a single application of either the intervention or placebo in the presence of trained pharmacy staff. Visit 2 will be triggered when the participant indicates via their online symptom diary that they have returned to normal skin (stage 7). This visit will involve confirmation of healing by pharmacists and checking for adverse events.

Participants will report symptoms and healing progression in an online daily diary (hosted on Castor EDC) which researchers will monitor for reports of adverse side effects. These diaries will also be used to monitor patient adherence to study requirements.

Participants will receive a follow-up phone call by a member of the research team 2 weeks after their final pharmacy visit to monitor any adverse events post treatment.

All data will be securely held on the study team database, accessible only by authorised investigators for the purposes of monitoring. The final dataset will be accessible by the coordinating investigators and each locality will retain on-site access to digital copies of source documentations with paper copies stored in secure archives.

Any changes to the study protocol are provided to the Human Research Ethics Comittee (HREC) and Health and Disability Ethics Committees as per protocol, trial registries will be updated and updated copies provided to trial sites. A copy of the consent form is included as online supplementary file 1 .

Clinical assessments and patient surveys will be completed using Castor clinical trials management software. All the pre-specified outcome measures are outlined in box 3 .

A systematic review by Harmenberg $e t a l,{ }^{1}$ a recent mini review by Hull ${ }^{13}$ and the most recent Cochrane systematic review protocol ${ }^{20}$ outline a number of important outcome measures which informed the choices in box 3 . Median duration of the episode, as defined by the time to healing either by the loss of the hard crust in participants with ulcerative lesions or the time to normal skin (complete epithelialisation) in those with aborted lesions, either participant or clinician reported is the most common clinically relevant outcome measure. However, both Harmenberg and Hull argue that using duration of healing alone may miss important clinical effects, for example, if lesion duration is less but the size of the lesion is greater this may not indicate an improvement in the condition from the patient's perspective. The ulcerative stage of the lesion is the most painful and cosmetically bothersome stage of the lesion. Given the difference in disease severity between ulcerative and non-ulcerative lesions, the proportion of these lesions is commonly used as another important outcome measure, which may be 
Box 3 Outcome measures

\section{Primary outcome measure:}

- Median episode duration in days (participant evaluated).

\section{Secondary outcome measures:}

- Median episode duration in days (investigator evaluated).

- Proportion (\%) of lesions progressing to ulceration.

- Median duration from wound development (stage 4) to healing (stage 7) in days (participant evaluated).

- NRS for pain, tenderness, tingling and itching during the symptomatic phase.

- Proportion (\%) of cases of HSV confirmed via swabs.

- Adverse event rate.

- Patient satisfaction and acceptability.

HSV, herpes simplex virus; NRS, numeric rating scale.

of greater concern to HSL sufferers than a small reduction of episode duration often seen in topical treatment. Harmenberg compellingly argues that an important marker in placebo-controlled trials is the proportion of participants that develop ulcerative lesions despite use of the study medication, as a reduction in progression to ulceration is of significant clinical benefit to sufferers. ${ }^{1}$ Indeed a common feature of antiviral therapies is not a significant reduction in overall duration, but a shortening of the duration of time of wound healing, ${ }^{21}$ which is still a very important clinical benefit.

Finally, the painful symptoms of burning, tingling and itching are commonly found in participants with HSL, and are usually bothersome and therefore a reduction in either severity or duration of these is an important patient-centric outcome.

\section{Participant evaluated lesion stage}

Participants will be provided with photo images as well as descriptions for each lesion stage via an online web form. We have used this lesion staging system in our previous trial. ${ }^{22}$ Participants will be asked to grade the lesion once per day, on waking, until return to normal skin occurs (stage 7) or until 14 days from pharmacy visit 1. A reminder SMS and/or email will be sent each morning with an embedded link to the web form. This grading will be submitted via an online web form. When a participant grades their lesion having resolved and the area has returned to normal skin (stage 7) via the online form they will be contacted to attend for assessment to their nearest study site within 24 hours by phone and email by the research assistant.

\section{Investigator-evaluated lesion stage}

Study participants will have their lesion stage graded by trained pharmacists and pharmacy technicians at two stages: (1) on the initial clinical visit for enrolment and (2) once participants indicate that healing has occurred they will have their lesion stage confirmed by clinicians at a study site within 24 hours (see exceptions below). Investigators will be provided with photo images as well as descriptions for each lesion stage, identical to those provided to the participant. They will assess this independently of the participant's own grading.

\section{Episode duration}

The episode duration will be defined as follows: if no vesicle formed then duration was from the initiation of treatment to the return to normal skin with cessation of signs or symptoms (aborted lesion); if a vesicle formed, the duration of episode was measured from the initiation of treatment to the loss of hard crust (stage 6: residual erythema could be present after loss of hard crust). Duration of wound healing will be determined by the time between the development of an ulcer (stage 4) to return to normal skin (stage 7). This will provide an estimate of the interventions effect on the most cosmetically bothersome and painful stage of the episode.

\section{Participant-graded pain and other symptoms}

Participants will grade lesion pain once per day on waking on a $0-10$ numeric rating scale (NRS) from onset of symptoms until healing occurs, with 0 being no pain and 10 the worse pain imaginable. Symptoms of burning, itching and tingling will be measured using a separate 4-point ordinal scale for each symptom as follows: $0=$ not present, $1=$ mild, $2=$ moderate and $3=$ severe. This score will be submitted via the online web portal along with lesion stage assessment.

\section{Participant satisfaction questionnaire}

At tpharmacy visit 2, participants will be asked to rate their satisfaction with the treatment given using a Likert scale. Questions will include the likelihood of recommendation of the intervention to family and friends, interest in using the intervention again for future cold sores and ranking the intervention relative to other previous treatments they have used (if any) for cold sores.

\section{Laboratory assessments}

For confirmation of HSV infection, participants will use the swab kits provided at the initial clinical consultation to take swabs of lesions that reach an ulcerative stage (stage 4) and do not have evidence of crusting (to avoid interference with the healing process). Samples will be taken by participants at home using a supplied dry swab that will be gently rubbed on the lesion in a circular motion for $5 \mathrm{~s}$, then placed in a sterile outer tube, stored in their fridge (below $8^{\circ} \mathrm{C}$ ) and shipped for viral culture to a central laboratory within 72 hours from sampling by courier. The presence of HSV will be diagnosed by the appearance of typical cytopathic effects. Isolates will be typed (HSV-1 and HSV-2) by immunofluorescent antibody technique. Those participants who have non-ulcerative (or aborted) lesions will not be asked to provide swabs as removing fluid can delay healing.

\section{Adverse events}

Adverse events will be monitored via the daily online diary, during the study visit 2 and 2 weeks after pharmacy visit 2 . 
Table 1 Timeline of treatment assessments and interventions

\begin{tabular}{|c|c|c|c|c|c|}
\hline Procedure & $\begin{array}{l}\text { Site visit } \\
1\end{array}$ & $\begin{array}{l}\text { Daily via online } \\
\text { diary }\end{array}$ & $\begin{array}{l}\text { If lesion reaches } \\
\text { stage } 4\end{array}$ & $\begin{array}{l}\text { Site visit } \\
2\end{array}$ & $\begin{array}{l}\text { Phone call } \\
2 \text { weeks after } \\
\text { site visit } 2\end{array}$ \\
\hline Written informed consent & $x$ & & & & \\
\hline Inclusion and exclusion & $x$ & & & & \\
\hline Medical history & $x$ & & & & \\
\hline Randomisation & $x$ & & & & \\
\hline First lesion occurs & $\mathrm{X}$ & & & & \\
\hline Clinical confirmation of lesion stage & $x$ & & & $\mathrm{X}$ & \\
\hline $\begin{array}{l}\text { Participant online diary-lesion stage, pain, } \\
\text { stage and other symptoms }\end{array}$ & & $x$ & & & \\
\hline Adverse events recorded & & $\mathrm{X}$ & & $\mathrm{X}$ & $\mathrm{X}$ \\
\hline Other treatments & & $\mathrm{X}$ & & & \\
\hline
\end{tabular}

HSV, herpes simplex virus.

Adverse events will be graded using the NCI Common Terminology Criteria for Adverse Events Version 4.0 and reported to the sponsor and HREC. After the end of study and locking of the study database, all adverse events will be summarised.

\section{Data monitoring and stopping guidelines}

Data will be monitored for completeness, plausibility and consistency to ensure the integrity and completeness of the dataset. Any queries will be resolved by the chief investigator or delegated member of the study team. Adverse events will be regularly monitored via the online daily diary, as well as at the pharmacy visit 2 and at the conclusion of the 2 week post trial period. Any serious adverse events will trigger an alert to the chief investigator.

\section{Study timeline}

The expected duration of the data collection phase of this study will be 12 months, with 14 time points where data will be collected. The schedule of events is outlined in table 1 .

\section{Statistical analysis of outcome data}

Efficacy analyses will be performed for both the intentto-treat population (all participants) and per-protocol population (excluding participants with missing data). The analysis for the primary outcome of median duration of episode as reported by the participant, from the stage at presentation to the clinical site until healing and for the secondary outcomes of the median investigator rated duration, and median duration of wound development will all be analysed using a Mann-Whitney $U$ test. Differences between groups in proportion of lesions progressing to ulceration, proportion of confirmed HSV, adverse events rates and participant satisfaction rates will be compared using $\chi^{2}$ tests.
Differences in NRS ratings for pain, tenderness, tingling and itching will be analysed using linear mixed model analysis of variance with group and time as fixed effects. In mixed model analysis subject will be used as a random effect. Secondary outcomes will include Cox proportional hazards for time to healing and time of wound duration and Kaplan-Meier survival plots. Multilevel analysis may be conducted with different factors including country, gender and number of previous episodes. Results will be documented with p-values and 95\% CIs. Data will be analysed using SAS V9.4 and/or SPSS V.24 software.

\section{Participant safety}

The product has been listed as a listed medicine on the Australian Register of Therapeutic Goods (ARTG) (AUST L 241934).

The Australian Therapeutic Goods Administration (TGA) have approved the safety of all ingredients for use in complementary medicines in Australia. A detailed dose justification and safety profile with supporting literature for each of the components of Dynamiclear are detailed in Product Information provided by the Sponsor, SciChem, in support of listing on the ARTG.

As the investigational product (Dynamiclear) is a lowrisk product already approved by the TGA and available for purchase, a formal data monitoring committee will not be established in Australia or New Zealand. However, the nominated medical representatives for the study, Dr Carolyn Ee (a registered General Practitioner in Australia) and Dr Alex Semprini (a registered medical practitioner in New Zealand), will review safety on all adverse events. The Dynamiclear formulation is considered safe to apply topically at the dosages outlined; however, individual components may irritate the skin in sensitive individuals. 
Copper sulfate, Calendula and Hypericum can all be irritating to the skin and mucosa in some individuals.

Methods for adverse event recording and reporting include the daily online diary which asks participants to report any adverse events over the previous 24 hours. Adverse events are also recorded at site visit 2 by the pharmacist and any post-trial events by a phone call 2 weeks following site visit 2. All participants will also be provided with a digital emergency contact card with details of whom to contact in the case of an emergency.

\section{Post-trial care}

After the trial has been completed and data analysis undertaken, participants will be advised of their group allocations and the study results. If the intervention is found to be effective, those in the placebo group will be offered one free treatment of the interventional product. All participants will be advised of the availability of the name of the product and its availability to purchase overthe-counter, if they wish to use it in future. Full indemnity insurance is in place for the study sponsor in the case of claims resulting from trial participation.

\section{Ethics, study registration and dissemination}

Results will be published in international academic journals and presented at conferences. Participating pharmacies will receive summarised results of the publication.

\section{Twitter Mike Armour @drmikenz}

Collaborators Members of the PRN in Australia (CBD Pharmacies Sydney, ChemistWorks Broadway, Priceline Pharmacy Oxford Street, Cincotta Discount Chemist Belrose, McFadden's Pharmacy St Ives, 0'Loughlin's Medical Pharmacy St Ives, Warringah Mall Pharmacy, Donworth Pharmacy, MediADVICE Pharmacy Doonside, MediADVICE Pharmacy Glenmore Park, MediADVICE Pharmacy St Clair, Priceline Pharmacy The Ponds, Figtree Plaza Chemist).

Contributors MA and AS designed and wrote the protocol. CE provided input related to medical components. MA and LM drafted the manuscript while AS, NS and CE provided critical edits and feedback on all versions of the manuscript. All authors reviewed the manuscript critically for important intellectual content, and approved the final manuscript for submission.

Funding This work was supported by Sci-Chem International Pty Ltd, Wetherill Park, NSW, Australia. Sci-Chem is the manufacturer of Dynamiclear. The Sponsor (Sci-Chem) was involved in the protocol design but has no role in the trial in any aspect of recruitment, intervention, data analysis or interpretation. The sponsor has no ability to modify or prevent manuscript publication on any trial-related outcomes.

Competing interests MA and AS received grant support from Sci-Chem International.

Patient consent for publication Not required.

Ethics approval Australian ethics approval from Western Sydney University Human Research Ethics Committee, ref: H12776. New Zealand Ethics approval from The Health and Disability Ethics Committees (HDEC), ref: 18/CEN/151.The trial was prospectively registered with the Australia New Zealand Clinical Trials Registry: ACTRN12618000890235. The Universal Trial Number (UTN) is U1111-1233-2426.

Provenance and peer review Not commissioned; externally peer reviewed.

Data availability statement Data sharing not applicable as no datasets generated and/or analysed for this study.

Open access This is an open access article distributed in accordance with the Creative Commons Attribution Non Commercial (CC BY-NC 4.0) license, which permits others to distribute, remix, adapt, build upon this work non-commercially, and license their derivative works on different terms, provided the original work is properly cited, appropriate credit is given, any changes made indicated, and the use is non-commercial. See: http://creativecommons.org/licenses/by-nc/4.0/.

\section{ORCID iDs}

Mike Armour http://orcid.org/0000-0001-7539-9851

Alex Semprini http://orcid.org/0000-0003-0949-0555

\section{REFERENCES}

1 Harmenberg J, Oberg B, Spruance S. Prevention of ulcerative lesions by episodic treatment of recurrent herpes labialis: a literature review. Acta Derm Venereol 2010;90:122-30.

2 Spruance SL, Overall JC, Kern ER, et al. The natural history of recurrent herpes simplex labialis. N Engl J Med Overseas Ed 1977;297:69-75.

3 Roizman B, Knipe D, Whitley R. Herpes simplex viruses. fields virology. 6th ED 2013:1897-8.

4 Baringer JR, Swoveland P. Recovery of herpes-simplex virus from human trigeminal ganglions. N Engl J Med 1973;288:648-50.

5 Perna JJ, Mannix ML, Rooney JF, et al. Reactivation of latent herpes simplex virus infection by ultraviolet light: a human model. $J$ Am Acad Dermatol 1987;17:473-8.

6 Scully C. Oral and maxillofacial medicine : the basis of diagnosis and treatment. 3rd ed. Edinburgh ; New York: Churchill Livingstone/ Elsevier, 2013.

7 Spruance SL, Bodsworth N, Resnick H, et al. Single-Dose, patientinitiated famciclovir: a randomized, double-blind, placebo-controlled trial for episodic treatment of herpes labialis. J Am Acad Dermatol 2006;55:47-53.

8 Leflore S, Anderson PL, Fletcher CV. A risk-benefit evaluation of aciclovir for the treatment and prophylaxis of herpes simplex virus infections. Drug Saf 2000;23:131-42.

9 Spruance SL, Nett R, Marbury T, et al. Acyclovir cream for treatment of herpes simplex labialis: results of two randomized, double-blind, vehicle-controlled, multicenter clinical trials. Antimicrob Agents Chemother 2002;46:2238-43.

10 Galeotti N, Vivoli E, Bilia AR, et al. St. John's wort reduces neuropathic pain through a hypericin-mediated inhibition of the protein kinase Cgamma and epsilon activity. Biochem Pharmacol 2010;79:1327-36.

11 Wölfle U, Seelinger G, Schempp CM. Topical application of St. John's wort (Hypericum perforatum). Planta Med 2014;80:109-20.

12 Nicolaus C, Junghanns S, Hartmann A, et al. In vitro studies to evaluate the wound healing properties of Calendula officinalis extracts. J Ethnopharmacol 2017;196:94-103.

13 Hull CM, Levin MJ, Tyring SK, et al. Novel composite efficacy measure to demonstrate the rationale and efficacy of combination Antiviral-Anti-Inflammatory treatment for recurrent herpes simplex labialis. Antimicrob Agents Chemother 2014;58:1273-8.

14 Dinda M, Mazumdar S, Das S, et al. The water fraction of Calendula officinalis Hydroethanol extract stimulates in vitro and in vivo proliferation of dermal fibroblasts in wound healing. Phytother Res 2016;30:1696-707.

15 Dinda M, Dasgupta U, Singh N, et al. Pi3K-Mediated proliferation of fibroblasts by Calendula officinalis tincture: implication in wound healing. Phytother Res 2015;29:607-16.

16 Shishkov S, Varadinova T, Panteva M, et al. Effect of complexes of zinc, cobalt and copper with D-Aminosugars on the replication of herpes simplex virus type 1 (HSV-1). Met Based Drugs 1997;4:35-8.

17 Clewell A, Barnes M, Endres JR, et al. Efficacy and tolerability assessment of a topical formulation containing copper sulfate and Hypericum perforatum on patients with herpes skin lesions: a comparative, randomized controlled trial. J Drugs Dermatol 2012;11:209-15.

18 Semprini A, Singer J, Braithwaite I, et al. Kanuka honey versus aciclovir for the topical treatment of herpes simplex labialis: a randomised controlled trial. BMJ Open 2019;9:e026201.

19 Castor Electronic Data Capture [program]. Amsterdam 2016.

20 Lee C, Chi C-C, Hsieh S-C, et al. Interventions for treatment of herpes simplex labialis (cold sores on the lips). Cochrane database of systematic reviews 2011.

21 Hull C. Acyclovir and hydrocortisone cream for the early treatment of recurrent cold sores. Virus Adaptation and Treatment 2011;3:1-6.

22 Semprini A, Singer J, Shortt N, et al. Protocol for a randomised controlled trial of $90 \%$ kanuka honey versus $5 \%$ aciclovir for the treatment of herpes simplex labialis in the community setting. BMJ Open 2017;7:e017766. 